\title{
PALABRAS PRONUNCIADAS POR EL DOCTOR EDGAR CORZO SOSA EN EL DÉCIMO ANIVERSARIO DE LA REVISTA CUESTIONES CONSTITUCIONALES REVISTA MEXICANA DE DERECHO CONSTITUCIONAL, EL 19 DE MAYO DE 2010, EN LAS INSTALACIONES DEL TRIBUNAL SUPERIOR DE JUSTICIA DEL DISTRITO FEDERAL
}

Doctor Edgar Elías Azar, magistrado presidente del Tribunal Superior de Justicia del Distrito Federal y Consejero presidente del Consejo de la Judicatura del Distrito Federal,

Doctor Diego Valadés, investigador en el Instituto de Investigaciones Jurídicas de la UNAM, ex-director de dicho Instituto e integrante del Colegio Nacional,

Doctor Juan Luis González Alcántara, magistrado del Tribunal Superior de Justicia del Distrito Federal y colega del Instituto de Investigaciones Jurídicas de la UNAM.

Doctor Mario Melgar Adalid, investigador en el Instituto de Investigaciones Jurídicas de la UNAM, Patrono de la Universidad Americana de Acapulco, y distinguido columnista en periódicos de Estados Unidos y México,

Licenciada y doctoranda Ángela Quiroga Quiroga, directora del Instituto de Estudios Judiciales del Tribunal Superior de Justicia del Distrito Federal, y colega del Instituto de Investigaciones Jurídicas de la UNAM.

Estimados juzgadores y demás funcionarios judiciales,

Queridos abogados,

Estimados alumnos,

Amigas y amigos todos,

Agradezco las palabras que se han vertido en torno a mi persona. Son demasiado bondadosas y no me veo reflejadas en ellas. Considero que están guiadas más bien por la amistad y el compañerismo que por la objetividad de las cosas. 
Quiero extender un especial agradecimiento a todas las personas que hicieron posible que encontráramos un pretexto para reunirnos. Sin su esfuerzo e interés esta presentación no hubiera sido posible.

Quiero atraer su atención en torno a tres puntos.

En el primero les comentaré cómo fue que surgió la idea de reunirnos hoy. En el segundo cómo fue que apareció el primer número de Cuestiones Constitucionales, y por último algunas pinceladas de lo que ha pasado en diez años.

Durante estos diez años, como responsable editorial de Cuestiones Constitucionales, me he mantenido en contacto con los autores. En algunas ocasiones de manera directa y presencial, en otras, en cambio, de forma indirecta y virtual, ya que la tecnología informática nos empuja drásticamente hacia esta última.

Uno de los autores que nos acompaña en esta ocasión, Mario Melgar Adalid, un buen día me envió un artículo relacionado con la Suprema Corte de Justicia de los Estados Unidos de Norteamérica y el presidente Barack Obama. Me pareció un artículo interesante, bien pensado y articulado, como todo lo que escribe Mario, e inmediatamente nos dimos a la tarea de iniciar el proceso editorial. Para fortuna de la revista, de Cuestiones Constitucionales, habíamos recibido otro artículo relacionado con el mismo tema, escrito desde Alemania y con otro punto de vista. Cuando coinciden dos artículos, como en esta ocasión, siempre intentamos que ambos sean publicados en el mismo número, ya que así el lector se beneficiará al obtener diferentes referencias sobre un mismo fenómeno.

Ambos artículos pasaron con éxito el proceso de dictamen y se publicaron en el mismo número 21 del décimo aniversario de Cuestiones Constitucionales, número que presentamos hoy.

Otro buen dia, al coincidir con Mario Melgar en un viaje a Guadalajara, Jalisco, comentamos, como era de esperarse, el contenido de Cuestiones Constitucionales, de los artículos allí publicados y de la coincidencia de los dos artículos que abordaban la Corte de Estados Unidos y su relación con el presidente Obama. (abro un paréntesis sólo para señalar que en un artículo periodístico reciente, Mario Melgar abordó nuevamente este tema, provocando a los lectores en torno al cambio de presidente de la Corte de México). Llegamos al convencimiento que sería conveniente la presentación de Cuestiones Constitucionales. El lugar, día y hora, 
lo estamos viviendo en estos precisos momentos, entre amigos. Gracias Mario.

En este mismo sentido, deseo comentarles que uno de los momentos más gratos que he tenido como director editorial ha sido cuando recibo una contribución académica de alguno de los integrantes del Comité Editorial, de ese órgano colegiado que da realce y prestigio a la revista. Así ha ocurrido, recientemente, con Jorge Miranda y con Mario Melgar, y hago votos porque así siga ocurriendo.

En lo que hace al segundo de los puntos, quiero comentarles la forma en que nació Cuestiones Constitucionales, hace 10 años. El primer número vio la luz en julio de 1999.

Antes de ello, el de la voz traía a cuestas una larga estancia en el extranjero, en donde periódicamente consultaba las revistas española, francesa o las italianas de derecho constitucional, pues en ellas encontraba los artículos más actuales del derecho constitucional, y sobre ellos siempre se armaban discusiones académicas interesantes entre los doctorandos y, si bien iba, con los profesores universitarios.

Cuando terminé mis estudios y regresé a México, al Instituto de Investigaciones Jurídicas de la UNAM, extrañé inmediatamente esta costumbre. Acudí a la Biblioteca a ver los números más recientes de las revistas europeas de derecho constitucional, pero no estaban los más recientes. Tampoco encontré doctorandos o investigadores jóvenes que tuvieran la costumbre de comentar los artículos recién publicados de tales revistas, ni siquiera por internet. Me encontraba en una situación de vacío jurídico.

La sensación de vacío que les comento me orilló a la siguiente conclusión: hacía falta una revista mexicana de derecho constitucional que provocara los comentarios de los juristas mexicanos, pero al mismo tiempo que sirviera de material valioso para los doctorandos, que los profesores pudieran comentar el artículo de reciente publicación, no como una reimpresión de algo que había aparecido en otro lugar, sino como una verdadera contribución a la ciencia del derecho constitucional.

Así nació, en su concepto, Cuestiones Constitucionales. Pero faltaba, lo que a todas las ideas, su materialización.

Otro buen día (como se darán cuenta ya son muchos buenos días los que he tenido y hoy es otro más) llegó hasta mi cubículo una persona que tenía la intención de contender por la dirección del Instituto. Platicamos de muchas cosas de interés académico, especialmente de la Biblioteca, 
de la cual me encontraba al frente en esos momentos. Al final de la charla amena que tuvimos, ya cuando el contendiente se retiraba, me atreví a externarle (lo fue una osadía, como podrán deducir enseguida) lo que tiempo atrás ya tenía en mente, le dije: "Diego, si llegas a ser director sería muy conveniente crear la revista mexicana de derecho constitucional".

Diego no hizo mayor gesto, se quedó pensativo y no recibí respuesta alguna. A decir verdad sentí como si las palabras se las hubiera llevado el viento, pero al mismo tiempo me sentí tranquilo, pues había externado una inquietud que daba vueltas por mi cabeza hacía tiempo ya.

El tiempo pasó, Diego llegó a la dirección del Instituto de Investigaciones Jurídicas, y otro buen día me llamó a su oficina. Me dijo más o menos lo siguiente:

"Edgar, ¿te acuerdas del proyecto aquél que me comentaste cuando fui a verte a tu oficina?

Por supuesto Diego.

Pues bien, ¿cuándo crees que podamos tener el primer número?”.

Las palabras, entonces, no se las había llevado el viento. Había cargado con ellas Diego Valadés. La osadía, también, obtuvo una respuesta contundente.

De esta manera, un año después de esa entrevista corta pero sustanciosa, luego de haber obtenido la información correspondiente a la creación de otras revistas especializadas en derecho constitucional, como la española, la francesa y las italianas, y luego de haber estudiado los requisitos que exigía el Conacyt para que las revistas ingresaran a su prestigioso índice, apareció el primer número de Cuestiones Constitucionales.

El compromiso de Diego con Cuestiones Constitucionales siempre ha estado presente. Cuidó la aparición de cada número como pocas personas, hizo que saliéramos siempre al inicio del periodo, no al final como era la tradición.

Hay una anécdota en la que me quiero detener, con la venia de Diego, por supuesto. En las palabras que escribió a manera de presentación del primer número de Cuestiones Constitucionales, aparecido fuera de las instalaciones de la Universidad que por aquellos momentos estaba injustamente cerrada, planteó lo que entonces llamó la "nómina de los problemas constitucionales del Estado contemporáneo", una lista que contenía 21 temas, cada uno de los cuales constituía una reflexión de diversos momentos constitucionales, considerando tanto los que habíamos vivido 
en México y en el mundo, pero haciendo una apuesta por los que serían preocupación en los años venideros.

Pues bien, quiero decirles que de los 21 temas lanzados por Diego, durante estos diez años hemos abordado 16 . No los leo porque sería extenderme demasiado. Enuncio, sin embargo, los cinco temas que se han quedado en el tintero, pero que seguramente en un futuro Cuestiones Constitucionales dará cuenta de ellos. No hemos abordado el tema relacionado con los "flujos financieros internacionales", no porque no tengan una repercusión constitucional, que la tienen como queda evidenciado en las Constituciones de Brasil, Honduras, Nicaragua y Portugal, sino porque no hemos entrado de lleno en este terreno.

El "federalismo-regionalismo" es otro de los temas que no han sido abordados directamente en Cuestiones Constitucionales, quizá porque hay pocos especialistas en este tema, o bien porque muchos lo abordan pero de manera refleja.

Los "organismos no gubernamentales" también constituyen otro tema sin respuesta doctrinal. No hemos entrado analizar las formas en que los diversos sistemas constitucionales los han integrado y regulado, quizá porque sigue siendo un tema bastante espinoso, en el que muchos consideran saldrán mal librados.

La "regulación de procesos científicos y clínicos", relacionados con la investigación científica que plantea problemas éticos y jurídicos, no ha sido abordado con suficiencia, y si acaso lo ha sido de manera tangencial. Cuestiones Constitucionales está llamada a dar reflejo en sus páginas de este tema indicado por Diego, en el cual hoy en día nos encontramos inmersos.

Finalmente, los "límites del derecho", como tema relacionado con la desobediencia civil y la flexibilidad en el acatamiento de la norma, o las excepciones a la aplicación de la ley, tampoco ha sido objeto de un artículo en especial.

Como ustedes pueden fácilmente advertir, las reflexiones expuestas por Diego hace diez años, han sido abordadas en su totalidad, lo que significa que el análisis realizado en su momento fue por demás realista y certero. Es más, me atrevo a indicar que nos hemos quedado rezagados en la "nómina", por tanto hay que alcanzarla cuanto antes, lo cual quizá podamos conseguir en buena medida con alguna aportación del mismo autor de la nómina, del mismo Diego. 
Por todas estas cuestiones, no puedo sino concluir que Diego y Cuestiones Constitucionales están, si me permiten, vinculados indisolublemente. Él es el materializador de las Cuestiones, pero también el ideólogo señero de lo Constitucional.

Diego, muchas gracias por todo el empeño vertido para hacer que Cuestiones Constitucionales tuviera un buen inicio. Gracias a ese gran empujón todavía tenemos combustible para otros años más.

En cuanto al tercero y último de los puntos que deseo abordar hoy, abusando de su paciencia (pero que tendrá una sabrosa recompensa al final), les resumo brevemente lo que ha pasado en estos diez años.

Por desgracia, algunos de los integrantes del Comité Editorial o del Comité Asesor se han quedado en el camino. A ellos les rindo un tardío pero sincero agradecimiento.

Durante estos años hemos visto el nacimiento de otras revistas especializadas en derecho constitucional, pero por desgracia también hemos visto su agonía y en ocasiones extinción. Recuerdo, como si fuera ayer, las palabras del maestro Héctor Fix Zamudio: "el problema no es el inicio sino continuar por lo menos otros tres años más".

Siempre las he tenido presente y ahora, a los diez años, considero que las puedo glosar. Una revista no existe sino hasta después de su tercer año y es un proyecto estable a partir de los cinco. Llegar a los diez significa una publicación consolidada, con visibilidad internacional y objeto de referencia obligada en varios temas.

En una reciente búsqueda, encontré que existen en el mundo alrededor de veinte revistas especializadas en derecho constitucional. Las hay en Estados Unidos y Canadá; en América Latina, aunque no en el número que deseáramos, y también en Europa y algunos países del continente asiático. Veinte revistas, muchas de las cuales no pasan de diez años, es una cantidad poco representativa si consideramos que todos los países tienen cuestiones constitucionales o de constitucionalidad.

Dentro de las revistas de derecho constitucional que nos quedan más cerca, una italiana (Quaderni Costituzionalli) apareció en 1981, lo mismo que la Revista Española de Derecho Constitucional. La revista francesa llegó casi una década después. Cuestiones Constitucionales una década después que la francesa. La decana de todas, si no me equivoco, es otra italiana Giurisprudenza Costituzionalle, que vio la luz en 1956, por lo que acaba de pasar sus 50 años, edad que guardando las debidas distan- 
cias también alcanzó el Boletín Mexicano de Derecho Comparado, otra de las publicaciones del Instituto de Investigaciones Jurídicas de nuestra Universidad Nacional.

Por lo anterior, pero por muchas otras cosas que no puedo comentar debido a la brevedad del tiempo, el hecho de llegar a los diez años de publicación ininterrumpida es algo que debe festejarse, pero no para regocijarse por lo hecho, lo cual se logra en un solo instante, sino para obtener una bocanada de aire fresco que nos haga pensar que Cuestiones Constitucionales estará presente muchos años más.

Y como ya llegamos a la mayoría de edad en materia de revistas, y ya constituimos un proyecto viable para las revistas más añejas, en los primeros días de julio, realizaremos la I Reunión de Revistas Especializadas en Derecho Constitucional, a la cual asistirán las publicaciones periódicas más prestigiosas del mundo, evento que tendrá lugar en las instalaciones del Instituto de Investigaciones Jurídicas de la UNAM. Sería verdaderamente motivo de especial orgullo que el magistrado presidente Edgar Elías Azar, quien nos acompaña en esta ocasión, nos distinguiera con su presencia.

Durante esta década de existencia, han sido múltiples las vicisitudes que se han presentado en dirección editorial de Cuestiones Constitucionales. Me acuerdo de una anécdota que ilustra bien lo que trato de decir, y de la cual uno de los protagonistas fue Diego Valadés.

Con ocasión de la presentación del primer número de Cuestiones Constitucionales en el edificio de la Barra Mexicana Colegio de Abogados (me da mucha alegría que algunos de quienes se encuentran hoy estuvieron hace diez años), asistieron el entonces director del Instituto de Investigaciones Jurídicas de la UNAM, Diego Valadés, y el presidente de la Suprema Corte de Justicia de la Nación, Genaro David Góngora Pimentel. En ese momento Diego Valadés hizo alusión a que el de la voz era un investigador de tiempo completo, a lo que Genaro Góngora, llegado el momento replicó: "pues no sé cómo puede ser investigador de tiempo completo en el Instituto si Edgar es mi asesor".

Inmediatamente retumbó una frase más: "pues habría que preguntarle a su esposa con cuanto tiempo se queda ella".

Lo que quiero subrayar con esta anécdota es que la vida profesional del director editorial de una publicación periódica puede tener mil derroteros, pero la publicación sólo tiene una exigencia: la constancia. 
Por eso, para que un proyecto sea viable debe contar con una dirección editorial continua, quizá no para toda la vida, pero sí para un periodo cierto que haga que el proyecto se consolide y sea completamente viable.

Agradezco al director del Instituto de Investigaciones Jurídicas de la UNAM, el doctor Héctor Fix Fierro, la inmerecida confianza que ha continuado brindándome al dejarme estar al frente de esta publicación.

Estos diez años en verdad han sido pocos. Quizá me ha ayudado a verlo así lo que llamo una trampa psicológica. Veo a Cuestiones Constitucionales como la tercera de mis hijas. En consecuencia, puede ser que en ocasiones la revista no salga como uno lo ha previsto, entonces hay que pensar que ello quizá sea producto de la juventud, de la inexperiencia y que los años la irán colocando en su lugar. Pero también hay que pensar que a una hija no se la deja algarete, arrumbada, y mucho menos se entrega al primero que le cierre un ojo. Se la cuida, se está con ella el tiempo que más se pueda, y cuando llegue el príncipe azul, entonces y sólo entonces será el momento de entregarla, para que siga otro camino, aunque siempre estaremos pensando en ella y cuando lo requiera la apoyaremos.

Como alguno de ustedes ha podido observar en el díptico que se repartió, Cuestiones Constitucionales tiene algunos datos estadísticos que es importante tomar en consideración. Durante estos diez años hemos recibido un total de 317 colaboraciones, las cuales se dividen de la siguiente manera: artículos 161, comentarios jurisprudenciales 45, comentarios legislativas 37 , reseñas bibliográficas 74 . O sea que tenemos un promedio de colaboraciones de $31.7 \%$ por año.

De ese gran total, $63.4 \%$, es decir 201 colaboraciones son de México, $15 \%$, esto es 48 de Latinoamérica, destacando Brasil con 14 documentos y Argentina con 12 .

De ese gran total también $20.4 \%$, esto es, 65 son de Europa, sobresaliendo España con 34 e Italia con 12 documentos.

Y de ese gran total $0.9 \%$ esto es 3, provienen de Estados Unidos.

Este último es un dato duro que obviamente indica un rumbo que va a tener que tomar en los próximos años Cuestiones Constitucionales.

Quiero agradecer muy especialmente al doctor Edgar Elías Azar, magistrado presidente del Tribunal Superior de Justicia del Distrito Federal y del Consejo de la Judicatura, el habernos honrado con su presencia. Sin su generosa actitud tampoco hubiéramos podido realizar este evento académico. Me queda muy claro el interés que ha puesto en las cuestiones 
académicas, no sólo en la presentación de ésta y muchas obras más sino en la realización de proyectos de gran alcance académico, como estudios de doctorado para los funcionarios judiciales, diplomados y otras actividades que ayudan al servidor judicial a tener una visión más completa del derecho.

En alguna ocasión escuché decir a un alto servidor judicial federal que el Poder Judicial no está para competir con las universidades y que lo académico nada tiene que ver con lo judicial. En lo personal no comparto esta visión porque la poca experiencia que tengo me ha hecho ver que la academia y la impartición de justicia están extremadamente vinculadas, a grado tal que en ocasiones no sé si quien hace una sentencia está haciendo un libro o bien quien hace un libro está resolviendo un punto jurídico relevante que ayudará a resolver una contienda jurídica.

Gracias magistrado presidente Elías Azar por habernos permitido compartir estos gratos momentos con el Poder Judicial del Distrito Federal.

Agradezco, igualmente, a la directora del Instituto de Estudios Judiciales, Ángela Quiroga Quiroga, mi directora, pues soy integrante del Comité Académico de dicho Instituto, todo el apoyo que nos brindó para la organización de este evento, así como la excelente moderación que ha realizado. Me gusta el compromiso que ha adquirido con la institución y con la formación de los servidores judiciales. Ángela, gracias y sabes que cuentas con mi apoyo, aunque siempre, en principio, te diga que no.

Por último, quiero agradecer la presencia del magistrado y doctor Juan Luis González Alcántara, con quien me unen lazos de amistad y compañerismo académico. Es una excelente persona en quien siempre he encontrado palabras de apoyo, llenas de bonomía, y con quien compartí gratos momentos cuando estaba al frente del Tribunal Superior de Justicia y del Consejo de la Judicatura del Distrito Federal. Es un apasionado de los libros y de todo lo académico. Muchas gracias Juan Luis.

A las distinguidas personalidades que hoy nos acompañan tanto del foro, como de la magistratura del Poder Judicial, distinguidos alumnos, amigos y demás personas que nos acompañan, les agradezco su presencia y su paciencia.

Larga vida a Cuestiones Constitucionales. 